\title{
Low concentrations of nitric oxide delay the differentiation of embryonic stem cells and promote their survival
}

\author{
JR Tejedo ${ }^{*, 1}$, R Tapia-Limonchi ${ }^{2}$, S Mora-Castilla ${ }^{1}$, GM Cahuana ${ }^{1}$, A Hmadcha ${ }^{2}$, F Martin ${ }^{1}$, FJ Bedoya ${ }^{1}$ and B Soria ${ }^{2}$
}

Nitric oxide (NO) is an intracellular messenger in several cell systems, but its contribution to embryonic stem cell (ESC) biology has not been characterized. Exposure of ESCs to low concentrations $(2-20 \mu \mathrm{M})$ of the NO donor diethylenetriamine NO adduct confers protection from apoptosis elicited by leukaemia inhibitory factor (LIF) withdrawal. NO blocked caspase 3 activation, PARP degradation, downregulation of the pro-apoptotic genes Casp7, Casp9, Bax and Bak1 and upregulation of the antiapoptotic genes Bcl-2 111, Bcl-2 and Birc6. These effects were also observed in cells overexpressing eNOS. Exposure of LIF-deprived mESCs to low NO prevented the loss of expression of self-renewal genes (Oct4, Nanog and Sox2) and the SSEA marker. Moreover, NO blocked the differentiation process promoted by the absence of LIF and bFGF in mouse and human ESCs. NO treatment decreased the expression of differentiation markers, such as Brachyury, Gata6 and Gata4. Constitutive overexpression of eNOS in cells exposed to LIF deprivation maintained the expression of self-renewal markers, whereas the differentiation genes were repressed. These effects were reversed by addition of the NOS inhibitor L-NMMA. Altogether, the data suggest that low NO has a role in the regulation of ESC differentiation by delaying the entry into differentiation, arresting the loss of self-renewal markers and promoting cell survival by inhibiting apoptosis.

Cell Death and Disease (2010) 1, e80; doi:10.1038/cddis.2010.57; published online 7 October 2010

Subject Category: Experimental Medicine

Embryonic stem cells (ESCs) retain their ability to self-renew and to differentiate into a great variety of cell types. The genes Oct4, Sox2, Nanog and Myc are key regulators essential for the maintenance of self-renewal. ${ }^{1}$ The differentiation of ESCs is accompanied by the downregulation of these transcription factors and upregulation of transcription factors involved in differentiation. ${ }^{2}$ Loss of Oct 4 causes inappropriate differentiation of ESCs into trophectoderm, whereas overexpression of Oct4 results in differentiation into primitive endoderm and mesoderm. ${ }^{3}$ Nanog regulates pluripotency mainly as a transcriptional repressor of downstream genes that are important for cell differentiation, such as Gata4 and Gata6. ${ }^{4}$ These factors form a tight transcriptional regulatory circuit that maintains ESCs in an undifferentiated state.

In addition, these transcription factors are switched on and off by external signals. ${ }^{5}$ Thus, leukaemia inhibitory factor (LIF) maintains the self-renewal state in $\mathrm{mESCs}$ through activation of the JAK/STAT pathway. ${ }^{6}$ The main downstream target of the LIF is c-myc, an early response gene that promotes the expression of the regulatory subunit of telomerase. ${ }^{7}$ LIF also activates c-Src tyrosine kinase, extracellular signal-regulated kinases (ERKs) and the phosphoinositide 3-kinase, which is relevant for the proliferation and survival of mESCs. ${ }^{8}$
However, LIF signalling is not sufficient to sustain selfrenewal, and additional signals are required to repress differentiation. ${ }^{9}$ It has been shown that IRS-1 expression is necessary for maintaining the self-renewal state in mESCs, and that this action is independent of Stat3 phosphorylation. ${ }^{10}$ Also, expression of the active form of Akt is sufficient to maintain the undifferentiated phenotype of these cells. ${ }^{11}$ These reports suggest that the phosphoinositide 3-kinase/Akt pathway is necessary for the maintenance of self-renewal in mESCs.

In hESCs, the maintenance of undifferentiated growth is LIF independent. In this regard, hESCs grown in feeder layer-free culture require a medium conditioned by fibroblast culture. In the absence of such conditioned medium, high concentrations of basic fibroblast growth factor (bFGF) and Noggin are necessary to maintain self-renewal. ${ }^{12}$ In fact, activation of the FGF pathway is necessary for the control of survival and proliferation in several cell types. ${ }^{13}$ Increases in MAP-Erk kinase/ERK and Akt phosphorylation downstream of bFGF have been reported to occur in hESCs. ${ }^{14}$ Moreover, bFGF can function in cooperation with insulin-like growth factor II to maintain hESC pluripotency. Although bFGF contributes to cell survival with little influence on apoptosis, insulin-like

\footnotetext{
${ }^{1}$ Andalusian Center for Molecular Biology and Regenerative Medicine (CABIMER)-University Pablo de Olavide, CIBERDEM, Seville, 41092, Spain and ${ }^{2}$ Andalusian Center for Molecular Biology and Regenerative Medicine (CABIMER)-Fundación Progreso y Salud, Seville, 41092, Spain

${ }^{*}$ Corresponding author: JR Tejedo, Av. Américo Vespucio s/n-Edif. CABIMER. Parque Científico y Tecnológico Cartuja 93. Seville 41092, Spain. Tel: + 34 954 467840; Fax: + 34954 461664; E-mail: juan.tejedo@ cabimer.es

Keywords: embryonic stem cell; differentiation; self-renewal; nitric oxide

Abbreviations: ESCs, embryonic stem cells; NO, nitric oxide; LIF, leukaemia inhibitory factor; bFGF, basic fibroblast growth factor; DETA-NO, diethylenetriamine nitric oxide adduct; GFP, green fluorescent protein; eNOS, endothelial nitric oxide synthase; iNOS, inducible nitric oxide synthase; PI3K, phosphoinositide 3-kinase; Akt, protein kinase b; GC/PKG, guanylate cyclase/protein kinase G; IGF I, insulin-like growth factor I; IGF II, insulin-like growth factor II; SSEA 1, stage-specific antigen 1; SSEA 4, stage-specific antigen 4; AP, alkaline phosphatase

Received 06.5.10; revised 28.7.10; accepted 10.8.10; Edited by A Finazzi-Agro'
} 
growth factor-II increases proliferation and prevents apoptosis. ${ }^{15}$ Other signal transduction pathways that have a role in hESC self-renewal include the transforming growth factor- $\beta$ (TGF-b)/Activin/Nodal, ${ }^{16,17}$ phosphoinositide 3-kinase ${ }^{18}$ and $\mathrm{Wnt}^{19}$ pathways.

Nitric oxide (NO) is a signalling molecule that regulates cell survival and proliferation in diverse cell types. ${ }^{20,21}$ It has also been shown to affect gene expression at the level of transcription and translation. ${ }^{22} \mathrm{NO}$ is produced during the development of organisms by isoforms of NO Synthases (NOSs), and it is feasible that it might contribute to the regulation of some features of ESC biology. It has been reported that at physiological concentrations, NO functions as a negative regulator of stem/progenitor cell proliferation, and it is critical for the initiation of differentiation. ${ }^{23} \mathrm{~A}$ distinct feature NO is that actions inside cells depend heavily on its concentration. In the micromolar or nanomolar range, activation of survival and of the guanylate cyclase/protein kinase G system has been reported. ${ }^{24}$ Homeostatic concentrations of NO $(1-10 \mu \mathrm{M})$ can initiate signalling pathways implicated in survival actions in RINm5F cells and promote the activation of c-Src, which at the same time induces the activation of IRS-1, phosphoinositide 3-kinase, AKT and eNOS as a part of the survival response. ${ }^{21}$ Moreover, the generation of NO participates in the activation of survival pathways by insulin-like growth factor- 1 and insulin in $\beta$-cells. ${ }^{25}$ Thus, many of the signalling pathways activated by NO in other cell systems are operative in ESCs. Recent reports have shown that a functional NO-cGMP signalling system might be involved in early differentiation events of ESCs. ${ }^{26}$ However, it has also been proposed that the dual role of NO in the control of ESC differentiation might depend on the expression of NO-signalling components rather than on the differential expression of NOS isoforms during this period of development. ${ }^{24,26}$

Great effort is being devoted to identifying strategies that improve the self-renewal and pluripotency of ESCs. Here, we provide evidence for a role of NO in delaying ESC differentiation. This effect is independent of LIF, suggesting that this messenger could be used to improve ESC self-renewal in culture.

\section{Results}

Differential expression of NO synthases and expression
of self-renewal markers in mESCs. NOS isoforms gene
expression analysis of D3-mESCs transfected with a construct
containing the eGFP gene under control of the Oct4 promoter
(D3-pOct4-eGFP-mESCs) and selected by sorting revealed
that cells cultured in the presence of LIF expressed the eNOS.
Differentiation of these cells following LIF withdrawal or as
embryoid bodies led to a substantial decrease of eNOS and
an increase in the expression of the iNOS isoform. Further,
nNOS expression remained unchanged except for a slight
increase in cells differentiated as embryoid bodies (Figure 1a).
A time course study revealed that eNOS expression levels
decreased significantly from the second day of LIF withdrawal;
the lowest levels were observed between 4 and 7 days
(Figure $1 \mathrm{~b}$ upper), whereas iNOS expression began on day 5
and peaked on day 7 (Figure $1 \mathrm{~b}$ lower).
Next, D3-pOct4-eGFP-mESCs were transfected with a vector that constitutively overexpresses the eNOS gene under control of the CMV promoter (D3-pOct4-eGFP-eNOSmESC). Both green mESCs were selected by sorting in control conditions (Figure 1c and, Supplementary Figure 1). These cells expressed Nanog, Oct4 and Sox2 more highly than did wild-type D3-mESCs. In addition, showed high levels of Tert (Figure 1c), telomerase activity (Figure 1d) and eNOS protein (Figure 1e) relative to D3-mESCs. Furthermore, colonies of these cells emit green fluorescence, as confirmed by fluorescence microscopy and flow cytometry (Supplementary Figure 1a and b).

Effect of NO on apoptosis induced by LIF withdrawal in mESCs. In D3-pOct4-eGFP-mESCs we studied the effect of low concentrations of the diethylenetriamine nitric oxide adduct (DETA-NO) on cell survival and apoptosis. LIF deprivation led to a 6.5-fold increase in DNA fragmentation (Figure 2a, shaded bars); the exposure to $2-10 \mu \mathrm{M}$ DETA-NO conferred protection against this action. DETA-NO concentrations of $20 \mu \mathrm{M}$ or higher induced a significant increase in apoptosis (3.5-fold at $20 \mu \mathrm{M}$ and eight-fold at $50 \mu \mathrm{M})$ in comparison to LIF control cells. Cells grown in the presence of LIF, a significant effect of DETA-NO on DNA fragmentation was observed at $50 \mu \mathrm{M}$, the highest concentration tested (Figure 2a, open bars). Next, we tested the effect of endogenously $\mathrm{NO}$ on cell survival by using D3-mESC-Oct4-eGFP-eNOS-mESCs (Figure 2b). A significant decrease in DNA fragmentation was observed in these cells when compared with D3 and D3-pOct4-eGFPmESCs cultured in the absence of LIF for 7 days. Overexpression of eNOS, however, did not completely prevent the apoptosis induced by LIF withdrawal. To identify the mechanism involved in the protective action of NO, we studied the expression of apoptotic genes in these cells (Figure 2c). D3-pOct4-eGFP-mESCs grown in the absence of LIF and in the presence of $2 \mu \mathrm{M}$ DETA-NO showed a decrease in the expression of pro-apoptotic genes, such as Bax, Bak1, Casp9 and Casp7. The expression levels of the anti-apoptotic genes Bcl2/11, Bcl2 and Birc6 were two-fold higher than the levels in cells cultured under LIF withdrawal conditions (Figure 2c). Moreover, PARP downregulation and caspase 3 activation induced by LIF withdrawal were inhibited by NO treatment (Figure 2d). In addition, R1-mESCs cultured in the absence of LIF and in the presence of $2 \mu \mathrm{M}$ DETA-NO displayed a distinct morphology when compared with cells cultured in the absence of LIF, some of them showing a compact morphology with well-defined borders typical of the 'stemness' state (Supplementary Figure 3).

Impact of NO on the regulation of pluripotency and differentiation markers in mESCs. A dose-dependency of DETA-NO was carried out to study the effect of NO on the pluripotency markers Oct4 and Nanog. Figure 3a shows that Oct4 expression was not affected by exposing cells to DETA-NO $(2-50 \mu \mathrm{M})$ in the presence of LIF, whereas Nanog expression was enhanced. Oct4 and Nanog expression decreased when cells were incubated with $50 \mu \mathrm{M}$ DETA-NO (Figure 3a). In mESCs cultured in the absence 
a

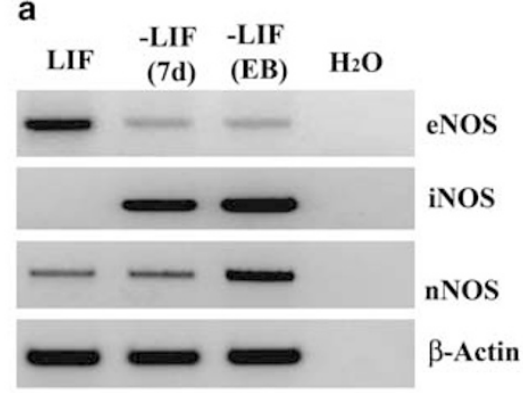

b
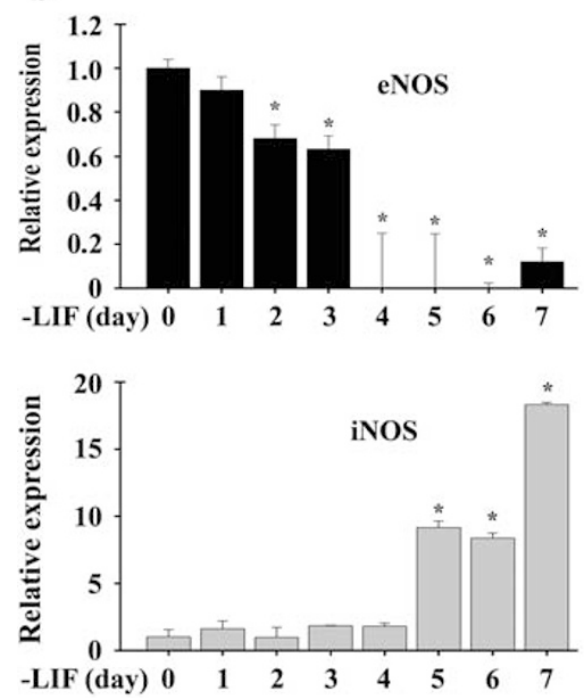

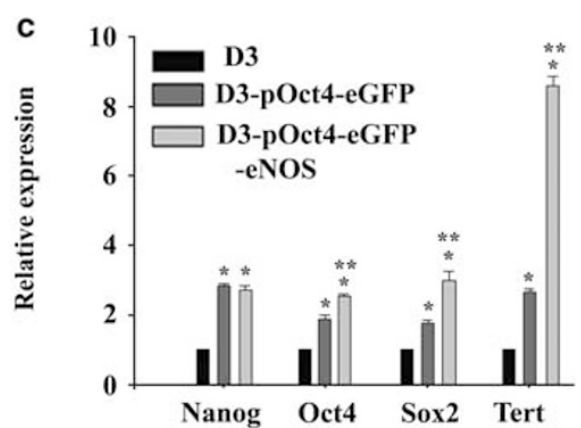

d

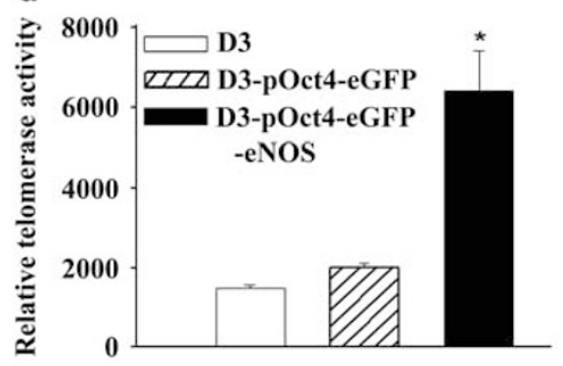

e

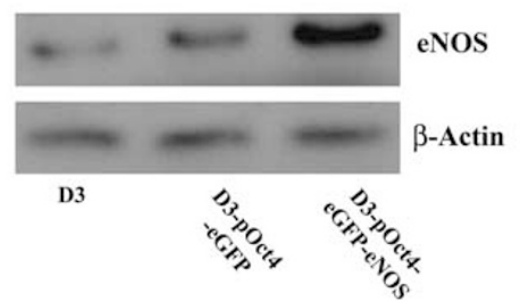

Figure 1 Differential expression of nitric oxide (NO) synthases and expression of self-renewal markers in mESCs. (a) NO synthase expression during differentiation. Sorted D3-pOct4-eGFP mESCs were cultured for 7 days in the presence or absence of LIF attached to a surface and for 14 days by hanging drop protocol (EB preparation). eNOS, iNOS and nNOS expression were detected by RT-PCR. The images are representative of three experiments. (b) Time course of eNOS and iNOS expression. R1-E mESCs were cultured in the absence of LIF for 7 days. eNOS and iNOS expression were detected by qRT-PCR, $\beta$-actin was used as an endogenous control and cycle threshold $\left(\mathrm{Ct}\right.$ ) values were normalized with respect to those of cells cultured in the presence of LIF (day 0 ). Data shown are from three independent experiments. ${ }^{*} P \leq 0.005$ with respect to day 0. (c) qRT-PCR of undifferentiation markers. D3 mESCs, D3-pOct4-eGFP mESCs and D3-pOct4-eGFP-eNOS mESCs were cultured in the presence of LIF and gene expression was determined by qRT-PCR. Data are the mean \pm S.E.M. of three experiments; ${ }^{*} P \leq 0.005$ when compared with $\mathrm{D} 3$ cells and ${ }^{* *} P \leq 0.005$ when compared with D3-pOct4-eGFP cells. (d) Telomerase activity in D3 mESCs, D3-pOct4-eGFP mESCs and D3-pOct4-eGFP-eNOS mESCs. Each data point represent the mean \pm S.E.M. of three experiments; ${ }^{*} P \leq 0.005$ when compared with other cell types. (e) Western blot analysis of eNOS in D3 mESCs, D3-pOct4-eGFP mESCs and D3pOct4-eGFP-eNOS mESCs. Representative image of three independent experiments

of LIF, DETA-NO $(2-50 \mu \mathrm{M})$ promoted both Oct4 and Nanog protein expression (Figure $3 \mathrm{a}$ ).

Using R1-E-mESCs we studied the effect of NO in other mESC line. R1-E-mESCs cultured in the presence of LIF grew in colonies displaying stronger positive staining for alkaline phosphatase $(100 \%)$ than cells grown in the absence of LIF (14\%). Supplementation of $2 \mu \mathrm{M} \mathrm{DETA-NO}$ to medium without LIF partially restored alkaline phosphatase activity (40\%); the number of positive colonies decreased as the concentration of DETA-NO increased: $5 \mu \mathrm{M}(36 \%), 10 \mu \mathrm{M}(25 \%), 20 \mu \mathrm{M}(20 \%)$ and $50 \mu \mathrm{M}(9.5 \%)$ (Figure $3 \mathrm{c}$ ). In addition, the expression of differentiation markers was analyzed by quantitative real time (qRT)-PCR. Early differentiation genes, such as Brachyury, Gata4, Gata6, Fgf5 and Fgf8 are expressed in cells cultured in the absence of LIF. DETA-NO supplementation led to lower expression of these genes (Figure $3 \mathrm{~b}$ ). The levels of expression observed with $2 \mu \mathrm{M}$ DETA-NO were comparable to those obtained in cells grown in the presence of LIF. Moreover, as the concentration of $\mathrm{NO}$ increased, the expression of differentiation markers also increased (Figure 3b).

NO delays mESC differentiation. We next investigated the effect of exogenous NO on differentiation. In D3-pOct4eGFP-mESCs, the exposure to LIF led to the expression of GFP in $69.7 \pm 8.5 \%$ of cells. However, when cells were cultured in the absence of LIF, the percentage of GFPpositive cells decreased $(27.7 \pm 5.5 \%)$. Supplementation with $2 \mu \mathrm{M}$ DETA-NO increased the percentage of GFP-positive cells $(45.4 \pm 6.5 \%)$ (Figure $4 a)$.

In addition, mESCs cultured in the absence of LIF supplemented with $2 \mu \mathrm{M}$ DETA-NO showed significantly higher expression of Oct4, Sox2 and Nanog than cells cultured in the absence of LIF (Figure 4b). Western blot 

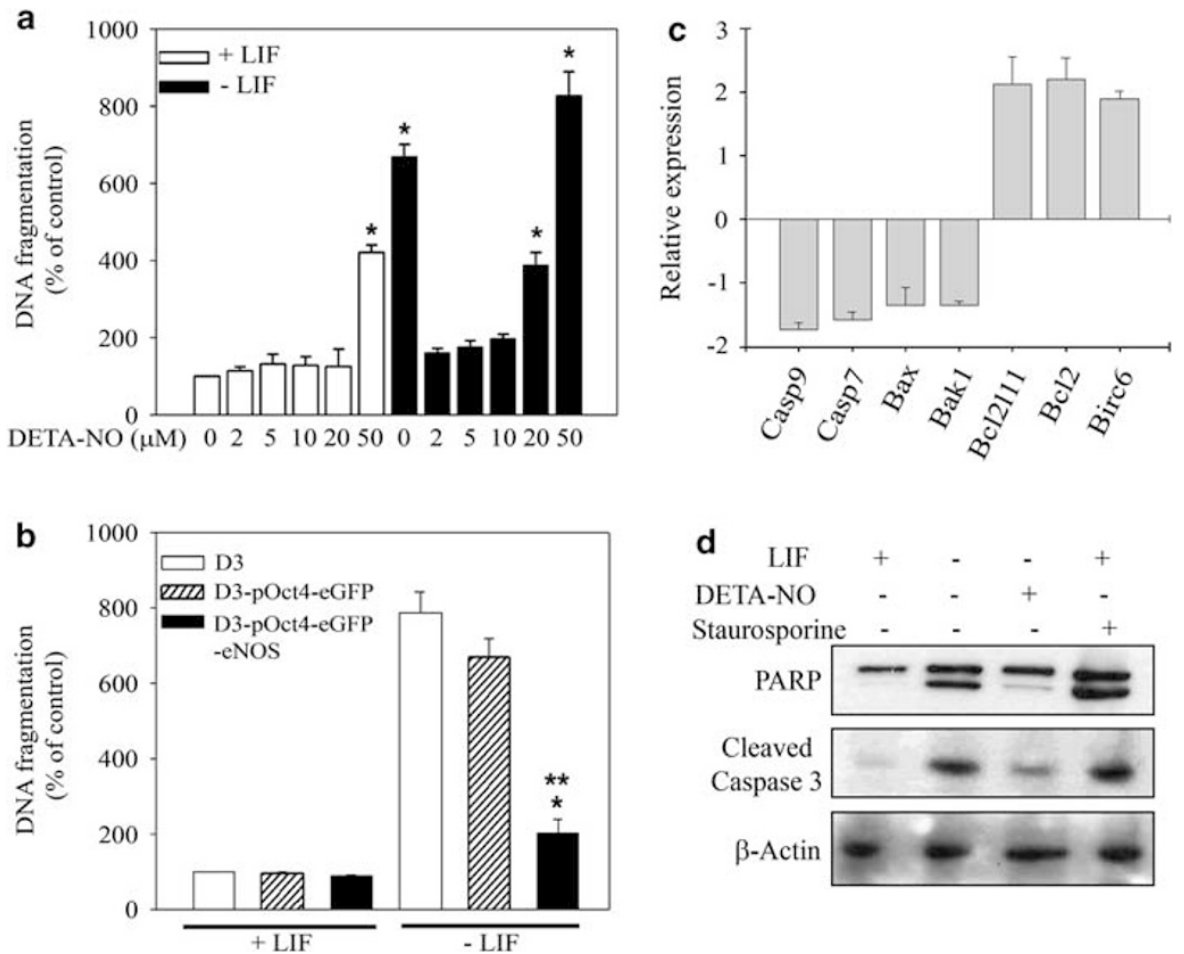

Figure 2 Effect of different NO concentrations on apoptosis and cell survival. (a) Effect of DETA-NO on DNA fragmentation. D3-pOct4-eGFP mESCs were cultured for 7 days in the presence or absence of LIF treated with different concentrations $(2-50 \mu \mathrm{M})$ of DETA-NO. DNA fragmentation was measured. Data are the mean \pm S.E.M. of five independent experiments. ${ }^{*} P \leq 0.005$ versus. D3 mESCs cultured in the presence of LIF and in the absence of DETA-NO (b) D3, D3-pOct4-eGFP and D3-pOct4-eGFP-eNOS mESCs were cultured in the presence or absence of LIF for 7 days and DNA fragmentation was measured. Data are the mean \pm S.E.M. of five independent experiments. ${ }^{*} P \leq 0.005$ versus. D3 and D3-pOct4-eGFP mESCs cultured in the absence of LIF. ${ }^{* *} P \leq 0.005$ versus. mESCs cultured in the presence of LIF. (c) Analysis of pro-apoptotic and anti-apoptotic gene expression in D3-pOct4-eGFP mESCs. Cells were cultured in the absence of LIF and treated with $2 \mu \mathrm{M}$ DETA-NO gene expression was determined by qRT-PCR. $\beta$-actin was used as an endogenous control and Ct values were normalized with respect to the Ct of cells cultured in absence of LIF. Data are the mean \pm S.E.M. of three experiments. (d) Effect of DETA-NO on PARP and caspase 3 cleavage. D3-pOct4-eGFP mESCs were cultured in the presence or absence of LIF for 7 days and supplemented with $2 \mu \mathrm{M}$ DETA-NO. PARP and cleaved caspase 3 were detected by western blot. The positive control for apoptosis was $1 \mu \mathrm{M}$ Staurosporine. Figures are representative of three independent experiments

analysis also showed higher levels of these proteins, but with Sox2 and Nanog in a different pattern than the gene expression. It is of note that the levels of the proteins shown were similar to those found in undifferentiated cells (Figure 4c). In non-transfected D3-mESCs, the expression of Brachyury (Figure 4d) increased by LIF-withdrawal was cancelled by $2 \mu \mathrm{M}$ DETA-NO. In addition, the co-expression of SSEA-1 and Oct4 protein (Figure $4 \mathrm{e}$ and Supplementary Figure 2A) decreased in the absence of LIF, whereas simultaneous treatment with DETA-NO maintained the Oct 4 and SSEA-1 signals. To address the effect of NO on apoptosis and pluripotency/differentiation, we monitored caspase 3 activation and Nanog and Brachyury expression in the presence of DETA-NO and staurosporine, an inducer of apoptosis (Supplementary Figure $2 \mathrm{C}$ ). Staurosporine cancels the protective effect of NO on apoptosis induced by LIF withdrawal without affecting the effect of $\mathrm{NO}$ on Nanog or Brachyury expression.

Endogenous eNOS overexpression maintains mESCs in an undifferentiated state. A second approach was to study the effect of endogenous NO production by eNOS overexpression using D3-pOct4-eGFP-eNOS-mESCs. Growth in the presence of LIF resulted in $70.9 \pm 3.1 \%$ GFP-positive cells. In the absence of LIF, the proportion of GFP-positive cells decreased to $51.8 \pm 9.1 \%$. The proportion of GFPpositive cells dropped to $16.4 \pm 5.8 \%$ when NOS activity was inhibited with $400 \mu \mathrm{M}$ L-NMMA (Figure 5a).

qRT-PCR revealed that the expression of pluripotency markers was lower in D3-pOct4-eGFP-eNOS-mESCs cultured in the absence of LIF in comparison with those grown in its presence, but their expression decreased when NOS activity was inhibited with $400 \mu \mathrm{M}$ L-NMMA (Figure 5b). A similar effect was observed in western blot analysis (Figure $5 \mathrm{c}$ ). The protein levels of Oct4 decreased significantly, whereas Sox2 also decreased, but this change was not significant. It is of note that the levels of Nanog did not change significantly between the two conditions (Figure $5 \mathrm{c}$ ). In contrast, when NOS activity was inhibited, the expression of the Brachyury gene increased with respect to its expression in cells cultured with LIF (Figure $5 \mathrm{~d}$ ). The co-expression of SSEA-1 and Oct4 protein decreased in the absence of LIF, and it was completely inhibited when NO production was blocked with $400 \mu \mathrm{M}$ L-NMMA (Figure $5 \mathrm{e}$ and Supplementary Figure 2B). In the presence of LIF, L-NMMA partially reduced Oct4 expression both in wild-type mESCs and mESCs overexpressing eNOS (Supplementary Figure 2D). 
a

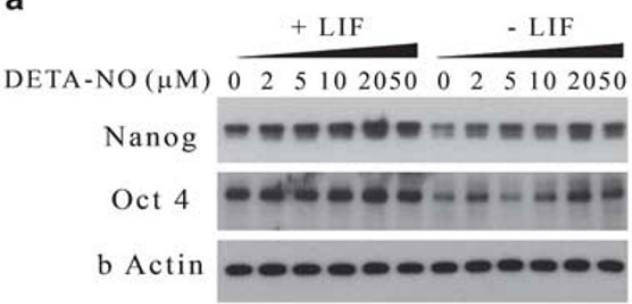

b
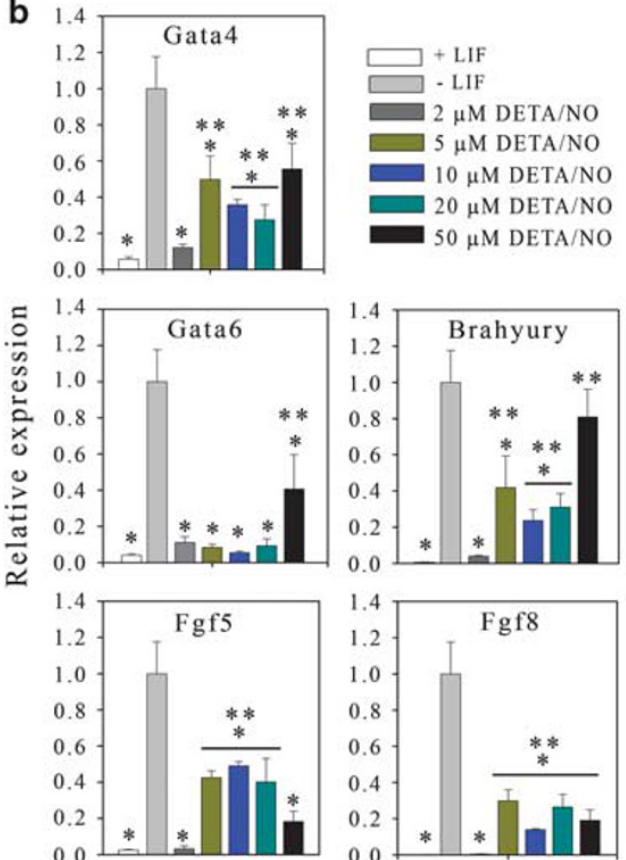
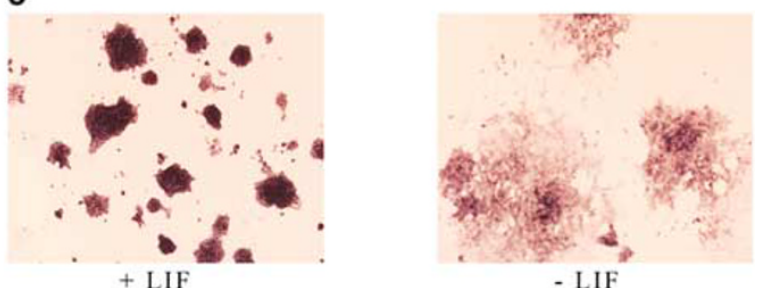

- LIF

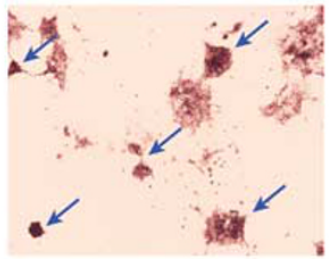

$2 \mu \mathrm{M} \mathrm{DETA} / \mathrm{NO}$

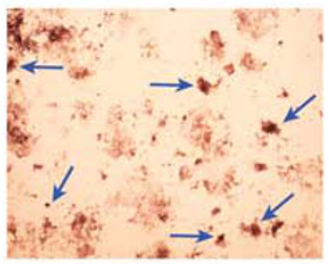

$10 \mu \mathrm{M}$ DETA/NO
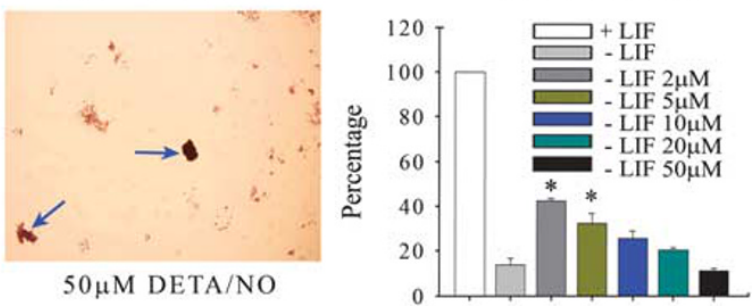

Figure 3 Dose-dependent influence of DETA-NO on pluripotency and differentiation in mESCs. (a) Western blot analysis of Nanog and Oct4 in non-transfected D3 mESCs cultured in the absence or presence of LIF and supplemented with different DETA-NO concentrations $(2-50 \mu \mathrm{M})$ over 7 days. The image is representative of five experiments. (b) Dose-dependent effect of DETA-NO on the expression of differentiation markers in R1-E mESCs cultured for 5 days; $\beta$-actin was used as the endogenous control and $\mathrm{Ct}$ values were normalized with respect to the $\mathrm{Ct}$ of cells cultured in absence of LIF. Data are the mean \pm S.E.M. of five independent experiments. ${ }^{*} P \leq 0.005$ versus. cells cultured in the absence of LIF. ${ }^{*} P \leq 0.005$ versus. cells cultured in the absence of LIF supplemented with $2 \mu \mathrm{M}$ DETA-NO. (c) Alkaline phosphatase activity retained by cells treated with different concentrations of DETA-NO. Percentages were obtained with respect to the number of colonies in the culture in presence of LIF. Scale bars, $20 \mu \mathrm{M}$. Figures are representative of three independent experiments. Percentage data are the mean \pm S.E.M. of three independent experiments. ${ }^{*} P \leq 0.005$ when compared with cells cultured in the absence of LIF

Low amounts of NO maintain hESC pluripotency. In the hESC HS181 line, the presence of $8 \mathrm{ng} / \mathrm{ml}$ bFGF maintained the undifferentiated features of colonies for up to five passages (Figure 6a, upper row, left picture). After five passages in the absence of bFGF, the colonies displayed white areas, which are typical of the differentiated state (Figure 5a, upper row, middle picture). When cells were cultured in bFGF-deprived conditions supplemented with $2 \mu \mathrm{M}$ DETA-NO for up to five passages, undifferentiated characteristics were restored and sub-culturing was possible for 10 passages (Figure 5a, upper row, right picture). In hESC colonies cultured for five passages, DETA-NO was observed to increase Oct4 expression, which was much greater than that noted under bFGF conditions (Figure $5 \mathrm{a}$, bottom row). Quantitative gene expression values were obtained for pluripotency markers and differentiation genes in hESCs. The expression of Nanog, Oct4 and Sox2 considerably declined in cells cultured in the absence of
bFGF; in contrast, cells treated with DETA-NO maintained higher levels of these mRNAs, yet still lower than cells cultured with bFGF (Figure 6b). These results were confirmed by western blotting (Figure 6c). By contrast, differentiation genes, such as Brachyury and Gata-4 showed a significant increase in expression in the absence of bFGF that was cancelled by addition of DETA-NO (Figure 6d). Next, we examined the expression of the cellsurface antigen SSEA-4. The results showed that SSEA-4 expression disappeared after five passages of bFGF withdrawal, but was present when the culture medium was supplemented with $2 \mu \mathrm{M}$ DETA-NO (Figure 6e).

\section{Discussion}

Evidence gathered in recent years indicates that NO participates in the regulation of cell differentiation and tissue morphogenesis, ${ }^{23,27}$ but its contribution to the control of ESC 


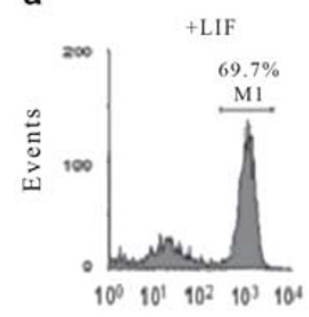

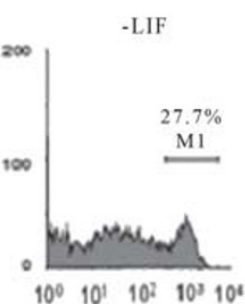

Fluorescence intensity (Arbitrary Units)

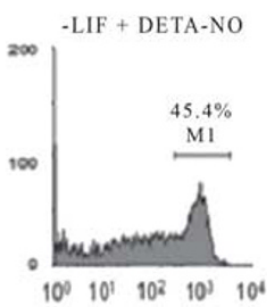

$\begin{array}{lllll}10^{0} & 10^{\prime} & 10^{2} & 10^{\prime} & 10^{4}\end{array}$ c
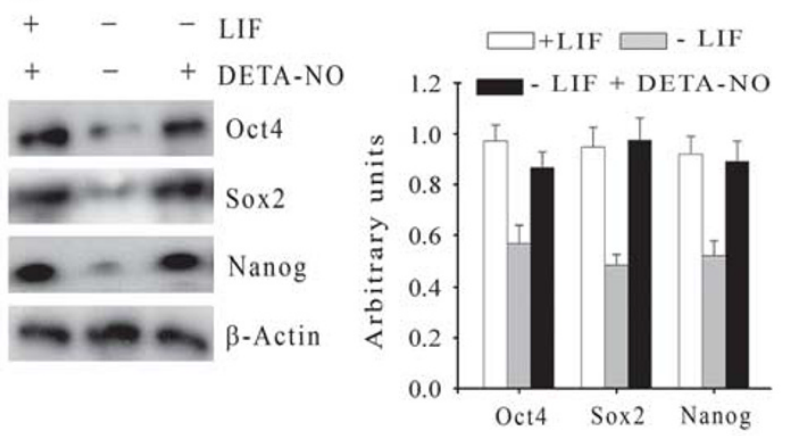

\section{b}
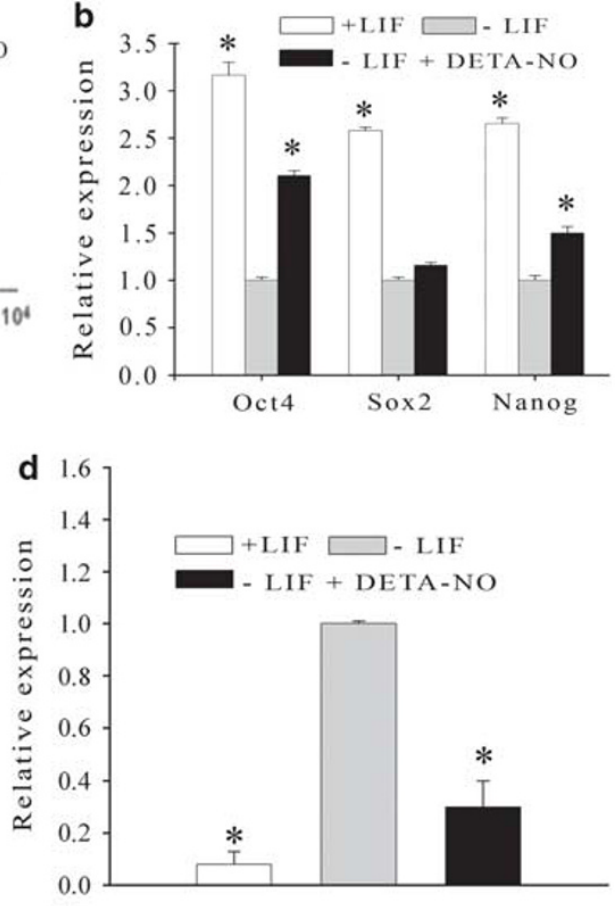

e

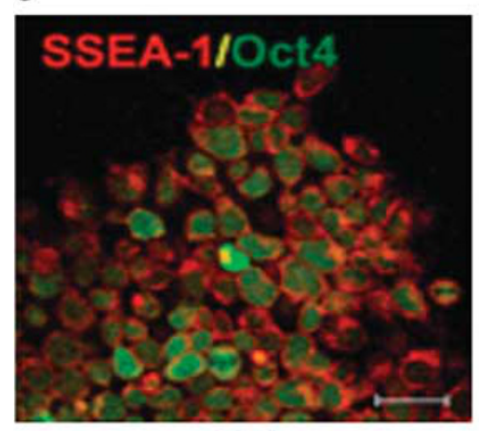

+LIF

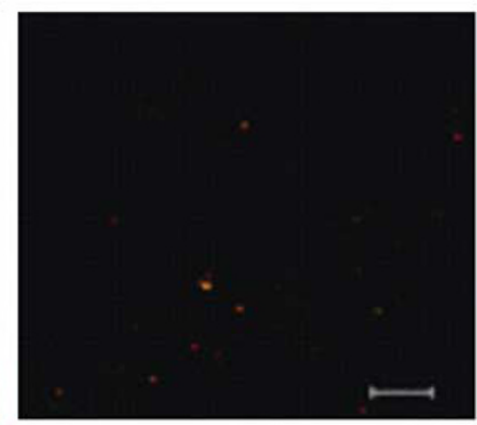

-LIF

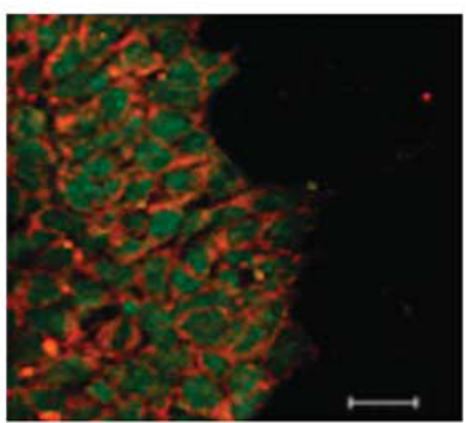

$-\mathrm{LIF}+2 \mu \mathrm{M}$ DETA-NO

Figure 4 Exogenous NO arrests the differentiation induced by LIF withdrawal in mESCs. (a) D3-pOct4-eGFP mESCs from passage 10 after sorting were cultured for 7 days in the presence (left panel) or absence (medium panel) of LIF and in the absence of LIF plus $2 \mu \mathrm{M}$ DETA-NO (right panel). GFP intensity was measured by flow cytometry. Cells with fluorescence intensity similar or higher than that observed for mESCs transfected with the pOct4-eGFP construct and cultured for 7 days in the presence of LIF were considered positive for eGFP (M1 population). Each graph is representative of five independent experiments $(n=5)$. (b) qRT-PCR analysis of Oct4, Sox-2 and Nanog in D3-pOct4-eGFP mESCs cultured for 7 days under the indicated conditions. $\beta$-actin was used as the endogenous control and Ct values were normalized with respect to the $\mathrm{Ct}$ of cells cultured in the absence of LIF. Data are the mean \pm S.E.M. of three experiments. ${ }^{*} P \leq 0.005$ versus. cells cultured in the absence of LIF. (c) Western blot analysis of Oct4, Sox-2 and Nanog in D3-pOct4-eGFP mESCs cultured for 7 days under the indicated conditions. Representative images and densitometry quantification are from five independent experiments. (d) qRT-PCR analysis of Brachyury expression in D3-pOct4-eGFP mESCs. $\beta$-actin was used as the endogenous control and Ct values were normalized with respect to the $\mathrm{Ct}$ of cells cultured in the absence of LIF. Data are the mean \pm S.E.M. of three experiments. ${ }^{*} P \leq 0.005$ versus. cells cultured in the absence of LIF. (e) Confocal images of cells positive for SSEA-1 in non-transfected D3 mESCs cultured for 7 days in the presence (left panel) or absence (middle panel) of LIF or in the absence of LIF plus $2 \mu \mathrm{M}$ DETA-NO (right panel). Nuclei are counter-stained with DAPI. Images are from three independent experiments. Scale bars are $20 \mu \mathrm{m}$

biology remained to be substantiated. Here, we show that low amounts of NO generated either from chemical donors or following eNOS overexpression regulate apoptosis, selfrenewal and differentiation of ESC. When mESCs were cultured under optimal conditions in the presence of LIF, high expression levels of eNOS and slightly lower levels of nNOS were detected. A specific decrease in the eNOS isoform was apparent when cells were exposed to one of two differentiation protocols, together with high expression of iNOS. The high expression of iNOS indicates that high levels of NO during sequential differentiation complement the effects induced by LIF withdrawal. High NO was previously shown to increase ESC differentiation towards definitive endoderm, cardiomyocytes and neurons. ${ }^{28-30}$

LIF deprivation induces also differential expression of NOS isoforms. eNOS expression significantly decreased after LIF withdrawal until day 6 , after which it maintained a low expression level, whereas iNOS expression began to increase after day 5 and was highest at day 7 . The level of expression of eNOS is associated to pluripotency as wild-type 

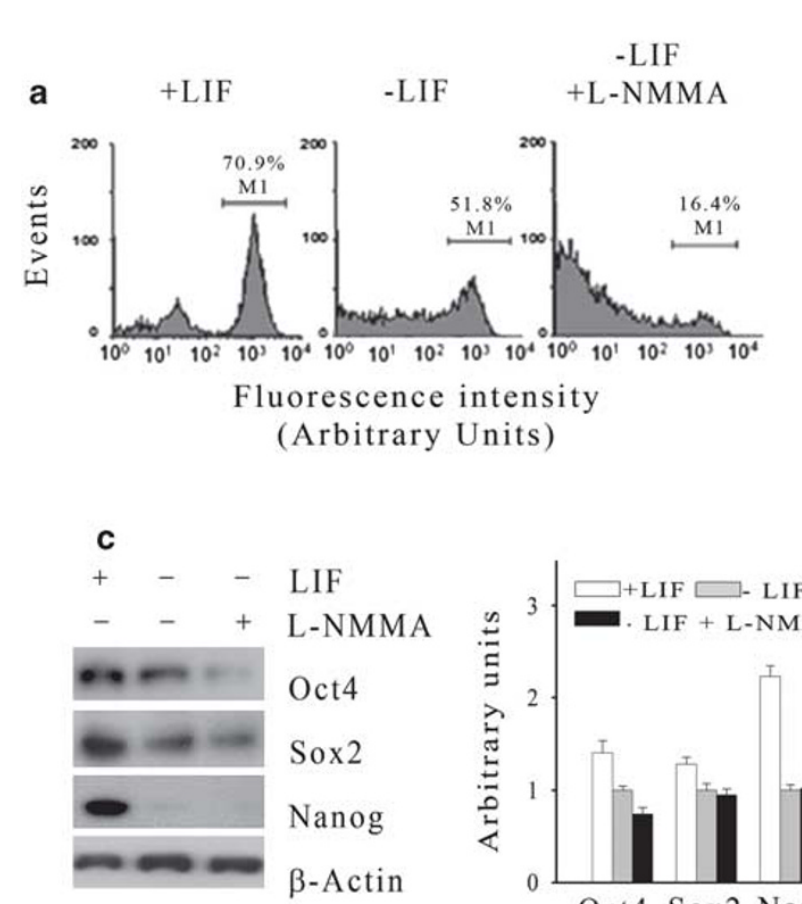

e

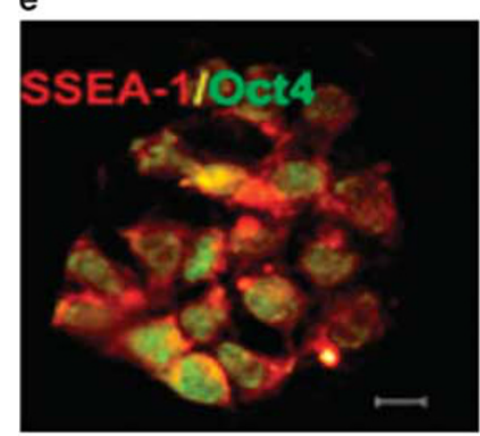

+ LIF
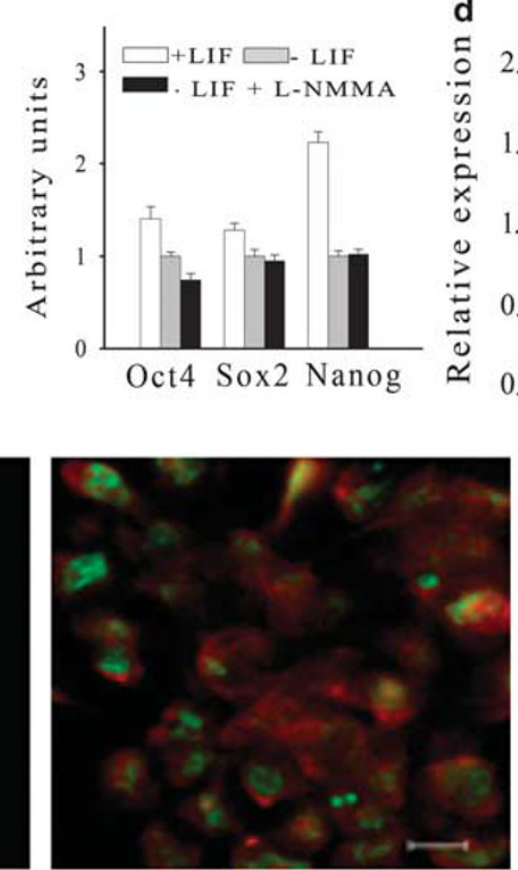

-LIF
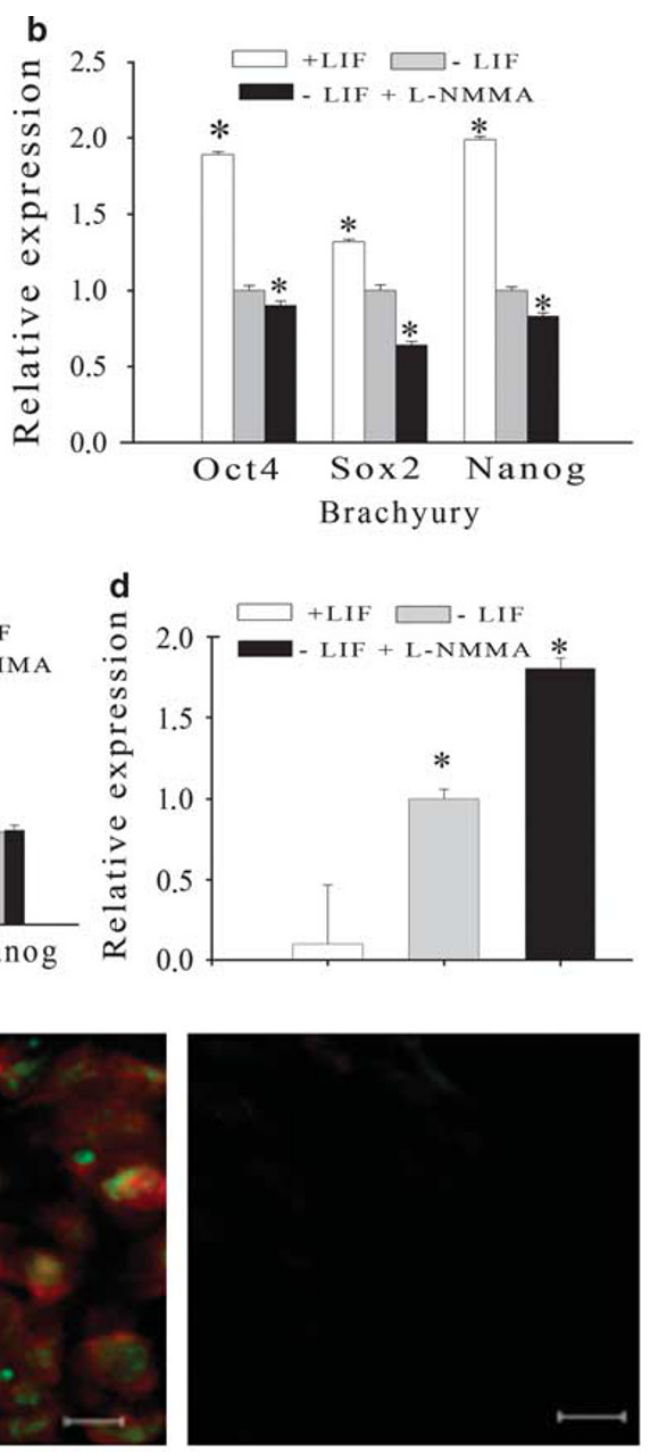

-LIF+L-NMMA

Figure 5 Endogenous production of NO by eNOS overexpression maintains mESC self-renewal after LIF withdrawal. (a) D3-pOct4-eGFP-eNOS mESCs from passage 10 after sorting were cultured for 7 days in the presence (left panel) or absence (medium panel) of LIF or in the absence of LIF plus $400 \mu$ M L-NMMA (right panel). GFP intensity was measured by flow cytometry. Cells with fluorescence intensity similar or higher than that observed for mESCs transfected with the pOct4-eGFP construct and cultured for 7 days in the presence of LIF were considered positive for eGFP (M1 population). Each graph is representative of five independent experiments $(n=5)$. (b) qRT-PCR analysis of Oct4, Sox-2 and Nanog in D3-pOct4-eGFP-eNOS mESCs cultured for 7 days under the indicated conditions. $\beta$-actin was used as the endogenous control and Ct values were normalized with respect to the $\mathrm{Ct}$ of cells cultured in the presence of LIF. Data are the mean \pm S.E.M. of three experiments. ${ }^{*} P \leq 0.005$ versus. cells cultured in the absence of LIF. (c) Western blot analysis of Oct4, Sox-2 and Nanog in D3-pOct4-eGFP mESCs cultured for 7 days under the indicated conditions. Representative images and densitometry quantification are from five independent experiments (d) qRT-PCR analysis of Brachyury expression in D3-pOct4-eGFP-eNOS mESCs. $\beta$-actin was used as the endogenous control and Ct values were normalized with respect to the Ct of cells cultured in the absence of LIF. Data are the mean \pm S.E.M. of three experiments. ${ }^{*} P \leq 0.005$ versus cells cultured in the presence of LIF. (e) Immunofluorescence images of cells positive for SSEA-1 in D3-pOct4-eGFP-eNOS mESCs cultured for 7 days in the presence (left panel) or absence (medium panel) of LIF or in the absence of LIF plus $400 \mu \mathrm{M} \mathrm{L-NMMA} \mathrm{(right} \mathrm{panel).} \mathrm{Nuclei} \mathrm{were} \mathrm{counter-stained} \mathrm{with} \mathrm{DAPI.} \mathrm{Images} \mathrm{are} \mathrm{representative} \mathrm{of}$ three independent experiments. Scale bars are $20 \mu \mathrm{m}$

D3-mESCs from passage 30 expressed lowed levels of eNOS, and low expression of Oct4, Nanog, Sox2, Tert and low telomerase activity. On the other hand sorted D3-pOct4eGFP-mESCs had increased levels of eNOS when compared with D3 wild-type cells and also had increased levels of Oct4, Nanog, Sox2, Tert and telomerase activity and eNOS overexpression significantly increased the expression of Oct4, Sox2, Tert and telomerase activity relative to D3-pOct4eGFP-mESCs. Furthermore, when eNOS activity was blocked, undifferentiation markers decreased notably. These findings support the notion that generation of low and sustained levels of NO might be instrumental for the maintenance of pluripotency. The hypothesis that precise cellular responses are differentially regulated by specific NO concentrations was previously proposed. ${ }^{31}$

Concentrations in the range $1-10 \mu \mathrm{M}$ of DETA-NO and endogenous NO generation by eNOS overexpression are also capable of halting apoptosis induced by LIF starvation. 
a
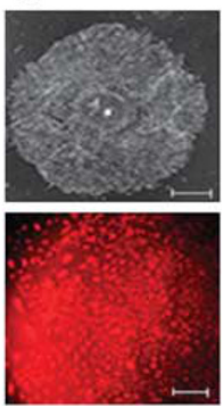

$+\mathrm{bFGF}$
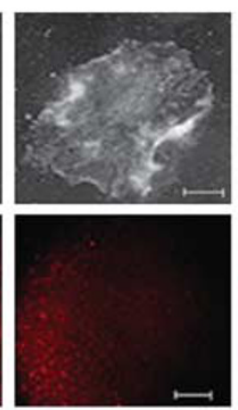

$-b F G F$
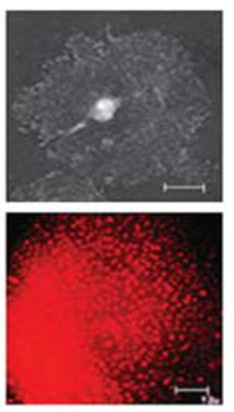

$-\mathrm{bFGF}$

+ DETA-NO

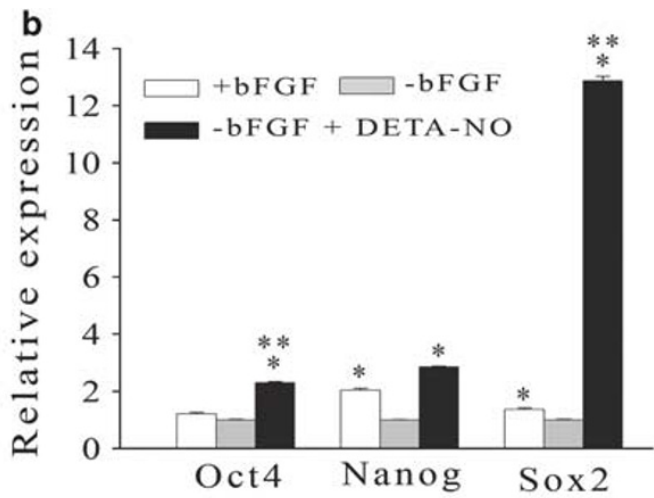

C

$+\quad-\quad$ bFGF

$+\quad+\quad+$ DETA-NO
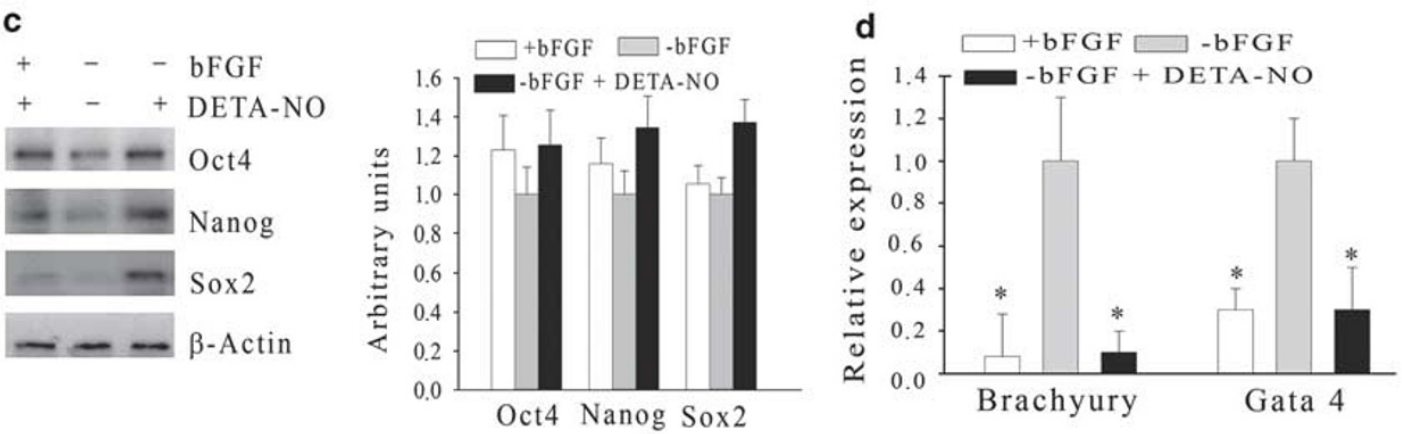

e

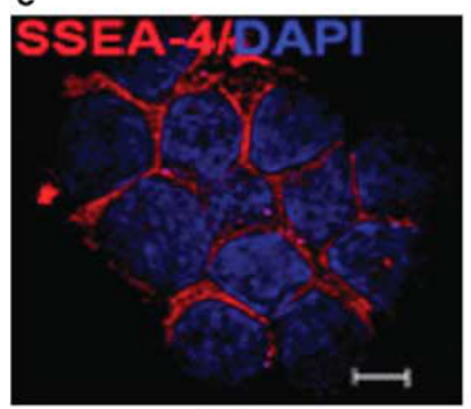

$+\mathrm{bFGF}$

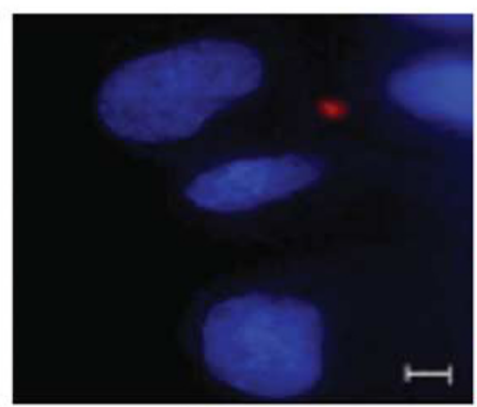

$-\mathrm{bFGF}$

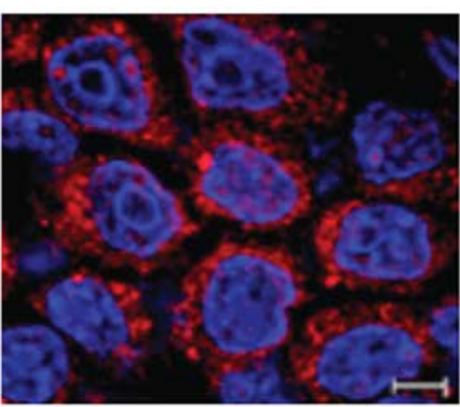

$-\mathrm{bFGF}$

+DETA-NO

Figure 6 Exogenous NO arrests the differentiation induced by bFGF withdrawal in hESCs cultured in a feeder-free system. (a) Upper panel: Representative phase contrast images showing the morphology of hESCs cultured for five passages in the presence (left) or the absence (middle) of bFGF and in the absence of bFGF plus $2 \mu \mathrm{M}$ DETA-NO (right). Images are representative of 10 experiments. Lower panel: immunofluorescence images of cells positive for Oct4 in hESCs cultured for five passages in the presence (left) or absence (middle) of bFGF or in the absence of bFGF plus $2 \mu \mathrm{M}$ DETA-NO (right). Images are representative of three experiments. Scale bars are $20 \mu \mathrm{m}$. (b) qRT-PCR analyses of Oct4, Sox-2 and Nanog in H181 hESCs cultured for 10 days under the indicated conditions. $\beta$-actin was used as the endogenous control and $\mathrm{Ct}$ values were normalized with respect to the $\mathrm{Ct}$ of cells cultured in the presence of bFGF. Data are the mean \pm S.E.M. of three experiments. ${ }^{*} P \leq 0.005$ versus cells cultured in the absence of bFGF. ${ }^{*} P \leq 0.005$ versus cells cultured in the presence of LIF. (c) Western blot analysis of Oct4, Sox-2 and Nanog in H181 hESCs cultured for 10 days under the indicated conditions. Representative images and densitometry quantifications are from five independent experiments. (d) qRT-PCR analysis of Brachyury and Gata4 expression in $\mathrm{H} 181 \mathrm{hESCs}$. $\beta$-actin was used as the endogenous control and Ct values were normalized with respect to the $\mathrm{Ct}$ of cells cultured in the absence of bFGF. Data are the mean \pm S.E.M. of three experiments. ${ }^{\star} P \leq 0.005$ versus cells cultured in the absence of bFGF. (e) Confocal images of cells positive for SSEA-4 in $h E S C s$ cultured for five passages in the presence (left panel) or absence (middle panel) of bFGF or in the absence of bFGF plus $2 \mu \mathrm{M}$ DETA-NO (right panel). Nuclei were counter-stained with DAPI. Images are representative of three experiments. Scale bars are $20 \mu \mathrm{m}$

Furthermore, treatment of mESCs with NO negatively regulates the pro-apoptotic response and notably increases the anti-apoptotic response. These results are consistent with previous reports in other cell models in which it was shown that NO prevented apoptosis by regulating $\mathrm{Bcl} 2$ family proteins. ${ }^{32}$ In addition, mESCs overexpressing Bcl2 maintain the characteristics of undifferentiated, pluripotent ESCs during long-term culturing and maintain their potential to differentiate into mature cell types. ${ }^{33}$ All these data are in agreement with other results describing the anti-apoptotic and survival effects of $\mathrm{NO} .^{20,21,25}$ A role for survival processes in the maintenance of the undifferentiated state in ESCs is also supported by several other studies..$^{8,11,14,15,18}$ In addition, using staurosporine, an inducer of apoptosis, together with 
DETA-NO, we showed that staurosporine cancels NO effect on caspase 3 activation induced by LIF withdrawal. In contrast, the effect of NO on Nanog and Brachyury levels was not altered. These results suggest that NO protective action is independent of its effect on pluripotency and differentiation.

The data presented in this report also provide evidence for a role for NO in ESC pluripotency. In the absence of LIF, a low level of DETA-NO maintains the self-renewal program in some colonies by enhancing the expression of self-renewal markers and increasing alkaline phosphatase activity. In addititon, both mESCs and hESCs cultured in the absence of LIF or bFGF and in the presence of NO, are capable of forming teratomas in immunosuppressed mice (Supplementary Figure 4). Previous reports have indicated a requirement for Nanog up-regulation for the maintenance of self-renewal. ${ }^{1,4,9,34}$ Masui et al. ${ }^{35}$ found that Sox2 controls the transcriptional activation of Oct4. In fact, it has been recently demonstrated that Oct4 expression is directly related to Sox2 expression. ${ }^{35,36}$ In accordance with these observations, our study showed that Oct4, Nanog and Sox2 expression levels are markedly increased by the addition of NO. Moreover, when eNOS activity is blocked, Sox2 expression decreases. One possibility is that NO could be controlling self-renewal and pluripotency by modulating Oct4 expression by increasing Sox2. Furthermore, in this context the relationship between eNOS expression and the maintenance of the undifferentiated state is relevant. In this regard, was observed that around $50 \%$ of D3-eNOS cells cultured in the absence of LIF failed to differentiate, whereas NOS inhibition by L-NMMA maintained only $16 \%$ of cells expressing GFP under control of the Oct4 promoter. Analysis of the gene expression and protein immunocytochemistry corroborated these results. It is interestingthat the protein levels did not correlate well with mRNA levels, suggesting that pluripotency marker regulation involves the regulation of protein levels. In this respect, roles for caspase activation and post-translational modification of self-renewal proteins have been recently put forward. ${ }^{5}$ It is important to note that the effects of $\mathrm{NO}$ on differentiation and the maintenance of pluripotency are dose dependent, with more beneficial effects at lower concentrations. Early differentiation markers, such as Brachyury, Gata 4, Gata6, Fgf5 and Fgf8 are considerably repressed.

The SSEA-1 surface marker of undifferentiated cells and colonies positive for alkaline phosphatase staining also showed that after five days of culture without LIF, several cells remained with markedly undifferentiated activity and morphology. Most of the cultured cells showed evidence of an initial differentiation process, suggesting that NO could be acting in a selective way on the total ESC population. This is in accordance with the notion that ESCs exhibit a high degree of heterogeneity reflected in their variegated expression of some of the pluripotency genes, promiscuous activation of lineagespecific genes and the fluctuating flow of differentiating cells in cultured populations. ${ }^{37}$

The mechanisms by which NO may modulate the differentiation of ESCs are a matter of debate. Studies on signal transduction pathways activated by $\mathrm{NO}$ could shed light on this issue. In fact, exposure to NO does not induce Stat3 phosphorylation in mouse or human ESCs (Supplementary Figure 5). This finding indicates that the mechanism of action of $\mathrm{NO}$ is independent of this pathway. This observation is consistent with reports showing regulation of Nanog that is independent from the LIF/Stat3 pathway ${ }^{4}$ and with the fact that Nanog over-expression in conditions of LIF deprivation is able to maintain self-renewal and increase the proliferation of ESCs. Moreover, the NO-induced activation of c-Src and Akt observed in ESCs (Supplementary Figure 5) is similar to our previous findings in other cell types. ${ }^{21}$ These results are also consistent with data reported by Anneren et al. ${ }^{38}$ and Rubbin et al., ${ }^{39}$ where they show that IRS-1 proteins, the PI-3K/Akt system and c-Src family proteins are important for $\mathrm{mESC}$ selfrenewal. However, the mechanisms involved in the regulation of these signalling pathways by NO need to be studied in greater detail.

Currently, diverse mechanisms are known that control the transition of ESC from self-renewal and the pluripotent state to differentiation. All of them involve the dynamic and intricate network of pluripotency and differentiation proteins, the first of which must be switched off to go ahead with differentiation. High amounts of NO control the mobilization and differentiation of various organ-derived precursor cells, probably by controlling the activation of promoter regions of some genes and by enforcing epigenetic changes involved in cell-cycle arrest and differentiation. ${ }^{22,40}$ We provide evidence for a role of low NO in controlling stem cell self-renewal and differentition. The nature of the events triggered in this novel process at the gene regulation level deserves further study.

\section{Materials and Methods}

Cell culture and generation of transfectants. Three embryonic stemcell lines were used: (i) mESC lines D3 and R1-E, obtained from the ATCC and (ii) hESC line HS181, kindly provided by the Karolinska Institute.

The D3 and R1-E mESCs were cultured at low density in Dulbecco's modified Eagle's Medium (Gibco, Carlsbad, CA, USA) supplemented with 15\% fetal bovine serum (Gibco), nonessential amino acids (1\%), 2-mercaptoethanol (0.1 mM), L-glutamine $(4 \mathrm{mM})$, sodium pyruvate $(1 \mathrm{mM})$, penicillin $(100 \mathrm{lU} / \mathrm{ml})$ and streptomycin $(0.1 \mathrm{mg} / \mathrm{ml})$. The undifferentiated state was maintained by adding $1000 \mathrm{U} / \mathrm{ml} \mathrm{rLIF} \mathrm{(Gibco)} \mathrm{to} \mathrm{the}$ culture medium. For the generation of transfectants, $\mathrm{mESC}$ s were electroporated at $800 \mathrm{mV} / 50 \mu \mathrm{F}$ with $30 \mathrm{ng}$ of linearized p-Oct4-eGFP-pgk hygro plasmid to obtain D3-pOct4-eGFP mESCs, and with $30 \mathrm{ng}$ of circular pCDNA3.1-eNOS (provided by Dr DJ Stewart, Department of Medicine, University of Toronto, Toronto, Ontario, Canada) to obtain D3-eNOS mESCs. Selection was initiated $48 \mathrm{~h}$ following transfection with $500 \mu \mathrm{g} / \mathrm{ml}$ hygromicin or $400 \mu \mathrm{g} / \mathrm{ml} \mathrm{G} 418$, respectively. Double transfectants (D3-pOCt4-eGFP-eNOS mESCs) were selected by the addition of the two antibiotics together. For experiments with mESCs, cells were cultured in colonies in the absence or presence of LIF. For treatments, increasing concentrations of DETA-NO were added to the culture medium of cells grown in the absence of LIF. The eNOS inhibitor L-NMMA was used at $400 \mu \mathrm{g} / \mathrm{ml}$.

hESCs were cultured on mitomycin C-inactivated human foreskin fibroblast feeder cells (CRL-2429, ATCC) at $37^{\circ} \mathrm{C}, 5 \% \mathrm{CO}_{2}$ in Dulbecco's modified Eagle's Medium/KO supplemented with 20\% knock-out serum replacement (Gibco Invitrogen Corporation, Carlsbad, CA, USA), $2 \mathrm{mM}$ L-glutamine (Gibco Invitrogen corporation), $0.1 \mathrm{mM}$ 2-mercaptoethanol (Gibco Invitrogen Corporation), 1\% nonessential amino acids (Gibco Invitrogen Corporation) and $100 \mathrm{U} / \mathrm{ml}$ per $100 \mathrm{mg} / \mathrm{ml}$ penicillin/streptomycin (Gibco Invitrogen Corporation). Cells were subcultured every 10 days by mechanical splitting. For the experimental conditions described here, hESCs were cultured on Matrigel-coated tissue culture plates (BD Biosciences, San Jose, CA, USA) in the previous medium in the absence or presence of $8 \mathrm{ng} / \mathrm{ml} \mathrm{bFGF}$ and $2 \mu \mathrm{M}$ DETA-NO.

RNA isolation and cDNA synthesis. Collected ESCs were washed with PBS $1 \times$ and then resuspended in Easy Blue reagent (Intron Biotechnology, Seoul, Korea) for RNA isolation. Following phenol/chloroform extraction, total RNA was precipitated in 2-propanol, washed with ethanol, suspended in nuclease-free water and stored at $-20^{\circ} \mathrm{C}$ until being used for $\mathrm{CDNA}$ synthesis. The absorbance of the total RNA for 
each sample was evaluated at 260 and $280 \mathrm{~nm}$ to determine its concentration and purity using a NanoDrop ND-1000 Spectrophotometer (Nanodrop, Willmington, DE, USA). Then, reverse transcription was performed with $1 \mu \mathrm{g}$ of RNA using $5 \mathrm{U} / \mu \mathrm{l}$ MMLV Reverse Transcriptase (Promega, Madison, WI, USA) and $12.5 \mu \mathrm{g} / \mu \mathrm{l}$ random primers (Promega) in a volume of $25 \mu \mathrm{l}$ and incubated for $1 \mathrm{~h}$ at $37^{\circ} \mathrm{C}$.

Quantification of gene expression by RT-PCR and telomerase activity. The primer sets are listed in Supplementary Table 1. The cDNA obtained was diluted 1: 10 and then used as a template for qRT-PCR using PerfeCTa SYBR GREEN Super Mix, Low ROX (Quanta Bioscience, Gaithersburg, MD, USA). Briefly, for a $20 \mu \mathrm{l}$ reaction: $10 \mu \mathrm{l}$ of SYBR Green master mix $2 X, 0.2 \mu \mathrm{M}$ primers, $1 \mu \mathrm{l}$ of diluted cDNA and water. Data collection was at $60^{\circ} \mathrm{C}, 1 \mathrm{~min}$ with the Applied Biosystems $7500 \mathrm{RT}$-PCR System (Applied Biosystems, Foster City, CA, USA). All the samples were normalized to the housekeeping gene $\beta$-actin, and all the data were analyzed with the $\Delta \Delta \mathrm{C}_{\mathrm{T}}$ method.

Telomerase activity was determined using the Telo TAGGG telomerase PCR

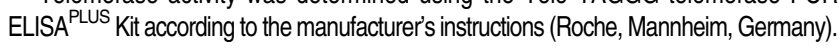

Flow cytometry and cell sorting. D3, D3-pOct4-eGFP and D3-pOCt4eGFP-eNOS mESCs were cultured for 7 days in the presence or in the absence of LIF and in the absence of LIF supplemented with $2 \mu \mathrm{M}$ DETA-NO or in the absence of LIF plus $400 \mu \mathrm{M}$ L-NMMA for D3-pOct4-eGFP-eNOS mESCs. Flow cytometry and cell sorting were performed using a FACSscan (BD Biosciences) and the data were analyzed using CELLQUEST software.

DNA fragmentation. DNA fragmentation was detected by an ELISA method according to the manufacturer's instructions (Roche).

Alkaline phosphatase assay. Cells were cultured at a density of $3 \times 10^{3}$ cells in $60 \mathrm{~mm}$ dishes for 5 days. Cells were fixed with $4 \%$ paraformaldehyde for $2 \mathrm{~min}$ at room temperature. Staining for alkaline phosphatase was performed using SIGMA FAST Alkaline Phosphatase Substrate Tablets Set (Sigma, St. Louis, MO, USA) following protocols provided by the manufacturer.

Immunofluorescence. mESCs were grown on Lab-Tek chamber slides (Nunc, Rochester, NY, USA), fixed with $4 \%$ paraformaldehyde and stained with mouse anti-Oct4 (1:200) (Chemicon, Charlottesville, VA, USA) and mouse anti-SSEA-1 (1:100) (Chemicon) primary monoclonal antibodies. hESCs were grown on Lab-Tek chamber slides coated with matrigel, fixed with $4 \%$ paraformaldehyde and stained with human anti-Oct4 (1:100) (Chemicon) and human anti-SSEA-4 (1:100) (Chemicon) primary monoclonal antibodies. Slides were incubated with anti-mouse immunoglobulin $\mathrm{M}(\mathrm{IgM})$ and anti-rabbit immunoglobulin $\mathrm{G}$ (IgG). Affinity-purified rhodamine red-conjugated antimouse IgM and fluorescein-conjugated anti-rabbit IgG (Jackson Immuno Research Laboratories, West Grove, CA, USA) were directed against the primary antibodies. Cells were counter-stained with $300 \mathrm{nM}$ DAPI. Slides and cover slips were mounted in a solution containing $4 \%$ propyl-gallacte (PN), $90 \%$ glycerol (VN) and $10 \%$ PBS (VN) and viewed with a Leica AF6000 microscope equipped with a fluorescent attachment or a Leica TCS SP5 confocal microscope. Images were processed with Metamorph.

Western blotting and immunoprecipitation. Total protein was extracted in RIPA buffer supplemented with protease and phosphatase inhibitors (Sigma). Protein concentrations were determined by BIO-RAD protein assay. Protein $(20 \mu \mathrm{g})$ was separated by $10 \%$ SDS-PAGE and transferred to a PVDF membrane (Amersham, GE Healthcare Europe GmbH, Barcelona, Spain). The membrane was incubated with primary monoclonal anti-mouse Oct4 $(1: 1000)$ (Bd Transduction Laboratories, San Jose, CA, USA), monoclonal anti-mouse c-Src (clone 327) (1:2000) (Chemicon), monoclonal anti-human Sox2 (1:1000) (R\&D Systems), polyclonal anti-mouse Nanog $(1: 2000)$ (Bethyl Laboratories, Montgomery, TX, USA), monoclonal anti-mouse $\beta$-actin $(1: 5000)$ (Sigma), polyclonal anti-human Stat3 $(1: 2000)$ (Upstate), monoclonal antimouse cleaved caspase-3 (Asp175) (1:1000) (Cell Signaling), polyclonal anti-mouse PARP $(1: 1000)$ (Cell Signaling), monoclonal anti-mouse pStat3 (Tyr 705) (1:1000) (Upstate), polyclonal anti-mouse Akt $(1: 2000)$ (Cell Signaling), polyclonal antimouse pAkt (Ser 473) (1:1000) (Cell Signalling), polyclonal anti-mouse pErk 1/2 (Thr202/Tyr204) (Cell Signalling) and polyclonal anti-human p-Src (Tyr 416) (1: 1000) (Cell Signalling). This incubation was followed by incubation with their respective secondary peroxidase-conjugated antibodies. Immunoreactive bands were detected by chemiluminescence reagents (Millipore, Billerica, MA, USA). For post-translational modification studies, the proteins were immunoprecipitated as described previously. ${ }^{21}$ Briefly, after treatment, mESCs and hESCs were lyzed in RIPA buffer (Sigma) supplemented with protease (Sigma) and phosphatase (Sigma) inhibitors for
$15 \mathrm{~min}$ at $4^{\circ} \mathrm{C}$. Then, lysates were centrifuged to remove debris, incubated with primary antibodies overnight at $4^{\circ} \mathrm{C}$ and finally incubated with Protein $\mathrm{G}$ Sepharose for $4 \mathrm{~h}$ at $4^{\circ} \mathrm{C}$. The beads were washed three times in lysis buffer and incubated with SDS-PAGE sample buffer for $10 \mathrm{~min}$ at $95^{\circ} \mathrm{C}$. The sample buffer was carefully removed and run on a 10\% SDS-PAGE gel and subsequently analyzed utilizing the phospho-specific antibodies pStat3 (Tyr 705), p-c-Src (Tyr 416), pErk 1/2 (Thr202/ Tyr204) and pAkt (Ser 473).

Statistical analyses. All data are the mean \pm S.E.M. of at least three independent experiments, except for the western blotting results, in which case a representative experiment is depicted in the figures. Comparisons between values were analyzed using one-way analysis of variance; $P \leq 0.05$ was considered statistically significant.

\section{Conflict of interest}

The authors declare no conflict of interest.

Acknowledgements. We thank the Karolinska Institute for providing the hESC lines. We also thank $Y$ Aguilera, N Mellado-Damas and AB Hitos for technical assistance and Centro Andaluz de Biología del Desarrollo for its support in the maintenance of mice. This study was supported by grants from Dirección General de Investigación Científica y Técnica (SAF2007/60105) and Junta de Andalucía (CTS576) to FJB; from Instituto de Salud Carlos III (TERCEL RD06/0010/0025), Fondo de Investigaciones Sanitarias (FIS-052106) and Dirección General de Investigación Científica y Técnica (CYT-836, SAF2005-08014) to BS; from Dirección General de Investigación Científica y Técnica (SAF2006-06673), Junta de Andalucía (exp. 0009/06, PI0022) and Instituto de Salud Carlos III (RETIC RD06/ 0015/0013 and CIBERDEM) to FM; from Consejería de Salud-Fundación Progreso y Salud (S0051) to A. Hmadcha; and from Consejería de Salud-Junta de Andalucia (PI-0095/2007) and Instituto de Salud Carlos III (CIBERDEM) to JRT.

1. Pan G, Thomson JA. Nanog and transcriptional networks in embryonic stem cell pluripotency. Cell Research 2007; 17: 42-49.

2. Liu N, Lu M, Tian X, Han Z. Molecular mechanisms involved in self-renewal and pluripotency of embryonic stem cells. J Cell Physiol 2007; 211: 279-286.

3. Niwa H. Molecular mechanism to maintain stem cell renewal of ES cells. Cell Struct Funct 2001; 26: 137-148.

4. Chambers I, Colby D, Robertson M, Nichols J, Lee S, Tweedie $S$ et al. Functional expression cloning of Nanog a pluripotency sustaining factor in embryonic stem cells. Cell 2003; 113: 643-655

5. Fujita J, Crane AM, Souza MK, Dejosez M, Kyba M, Flavell RA et al. Caspase activity mediates the differentiation of embryonic stem cells. Cell Stem Cell 2008; 2: 595-601.

6. Kristensen DM, Kalisz M, Nielsen JH. Cytokine signalling in embryonic stem cells. Apmis 2005; 113: 756-772.

7. Cartwright $P$, McLean $C$, Sheppard A, Rivett D, Jones K, Dalton S. LIF/STAT3 controls ES cell self-renewal and pluripotency by a Myc-dependent mechanism. Development (Cambridge, England) 2005; 132: 885-896.

8. Paling NR, Wheadon H, Bone HK, Welham MJ. Regulation of embryonic stem cell self-renewal by phosphoinositide 3-kinase-dependent signaling. The Journal of Biological Chemistry 2004; 279: 48063-48070.

9. Chen L, Yang M, Dawes J, Khillan JS. Suppression of ES cell differentiation by retinol (vitamin A) via the overexpression of Nanog. Differentiation; research in biological diversity 2007; 75: 682-693.

10. Rubin R, Arzumanyan A, Soliera AR, Ross B, Peruzzi F, Prisco M. Insulin receptor substrate (IRS)-1 regulates murine embryonic stem ( $\mathrm{mES}$ ) cells self-renewal. J Cell Physiol 2007; 213: 445-453

11. Watanabe $\mathrm{S}$, Umehara $\mathrm{H}$, Murayama $\mathrm{K}, \mathrm{Okabe} \mathrm{M}$, Kimura $\mathrm{T}$, Nakano T. Activation of Akt signaling is sufficient to maintain pluripotency in mouse and primate embryonic stem cells. Oncogene 2006; 25: 2697-2707.

12. Xu RH, Peck RM, Li DS, Feng X, Ludwig T, Thomson JA. Basic FGF and suppression of BMP signaling sustain undifferentiated proliferation of human ES cells. Nat Methods 2005; 2: $185-190$.

13. Bottcher RT, Niehrs C. Fibroblast growth factor signaling during early vertebrate development. Endocr Rev 2005; 26: 63-77.

14. Li J, Wang G, Wang C, Zhao Y, Zhang H, Tan Z et al. MEK/ERK signaling contributes to the maintenance of human embryonic stem cell self-renewal. Differentiation; research in biological diversity 2007; 75: 299-307.

15. Bendall SC, Stewart MH, Menendez P, George D, Vijayaragavan K, Werbowetski-Ogilvie T et al. IGF and FGF cooperatively establish the regulatory stem cell niche of pluripotent human cells in vitro. Nature 2007; 448: 1015-1021. 
16. Greber $\mathrm{B}$, Lehrach $\mathrm{H}$, Adjaye J. Fibroblast growth factor 2 modulates transforming growth factor beta signaling in mouse embryonic fibroblasts and human ESCs (hESCs) to support hESC self-renewal. Stem cells (Dayton, Ohio) 2007; 25: 455-464.

17. James D, Levine AJ, Besser D, Hemmati-Brivanlou A. TGFbeta/activin/nodal signaling is necessary for the maintenance of pluripotency in human embryonic stem cells Development (Cambridge, England) 2005; 132: 1273-1282.

18. Pyle AD, Lock LF, Donovan PJ. Neurotrophins mediate human embryonic stem cell survival. Nat biotechnol 2006; 24: 344-350.

19. Cai L, Ye Z, Zhou BY, Mali P, Zhou C, Cheng L. Promoting human embryonic stem cell renewal or differentiation by modulating Wnt signal and culture conditions. Cell Res 2007; 17: $62-72$.

20. Culmsee C, Gerling N, Landshamer S, Rickerts B, Duchstein HJ, Umezawa K et al. Nitric oxide donors induce neurotrophin-like survival signaling and protect neurons against apoptosis. Mol Pharmacol 2005; 68: 1006-1017.

21. Tejedo JR, Cahuana GM, Ramirez R, Esbert M, Jimenez J, Sobrino F et al. nitric oxide triggers the phosphatidylinositol 3-kinase/Akt survival pathway in insulin-producing RINm5F cells by arousing Src to activate insulin receptor substrate-1. Endocrinology 2004; 145: 2319-2327.

22. Contestabile A. Regulation of transcription factors by nitric oxide in neurons and in neuralderived tumor cells. Prog Neurobiol 2008; 84: 317-328.

23. Peunova N, Enikolopov G. Nitric oxide triggers a switch to growth arrest during differentiation of neuronal cells. Nature 1995; 375: 68-73.

24. Krumenacker JS, Katsuki S, Kots A, Murad F. Differential expression of genes involved in cGMP-dependent nitric oxide signaling in murine embryonic stem (ES) cells and ES cell-derived cardiomyocytes. Nitric Oxide 2006; 14: 1-11

25. Cahuana GM, Tejedo JR, Hmadcha A, Ramirez R, Cuesta AL, Soria B et al. Nitric oxide mediates the survival action of IGF-1 and insulin in pancreatic beta cells. Cell Signal 2008; 20: $301-310$

26. Mujoo K, Krumenacker JS, Wada Y, Murad F. Differential expression of nitric oxide signaling components in undifferentiated and differentiated human embryonic stem cells. Stem Cells and Development 2006; 15: 779-787.

27. Enikolopov G, Banerii J, Kuzin B. Nitric oxide and Drosophila development. Cell Death Differ 1999; 6: 956-963.

28. Mora-Castilla S, Tejedo JR, Hmadcha A, Cahuana GM, Martin F, Soria B et al. Nitric oxide repression of Nanog promotes mouse embryonic stem cell differentiation. Cell Death Differ 2010; 17: 1025-1033.

29. Kanno S, Kim PK, Sallam K, Lei J, Billiar TR, Shears II LL. Nitric oxide facilitates cardiomyogenesis in mouse embryonic stem cells. Proceedings of the National Academy of Sciences of the United States of America 2004; 101: 12277-12281.
30. Ciani E, Severi S, Contestabile A, Bartesaghi R, Contestabile A. Nitric oxide negatively regulates proliferation and promotes neuronal differentiation through N-Myc downregulation. J Cell Sci 2004; 117 (Pt 20): 4727-4737.

31. Thomas DD, Ridnour LA, Isenberg JS, Flores-Santana W, Switzer CH, Donzelli S et al. The chemical biology of nitric oxide: implications in cellular signaling. Free radic Biol Med 2008; 45: 18-31.

32. Chung HT, Pae HO, Choi BM, Billiar TR, Kim YM. Nitric oxide as a bioregulator of apoptosis. Biochem Biophys Res Commun 2001; 282: 1075-1079.

33. Yamane T, Dylla SJ, Muiitiens M, Weissman IL. Enforced Bcl-2 expression overrides serum and feeder cell requirements for mouse embryonic stem cell self-renewal. Proceedings of the National Academy of Sciences of the United States of America 2005; 102: 3312-3317.

34. Pan G, Pei D. The stem cell pluripotency factor NANOG activates transcription with two unusually potent subdomains at its $C$ terminus. J Biol Chem 2005; 280: 1401-1407.

35. Masui S, Nakatake Y, Toyooka Y, Shimosato D, Yagi R, Takahashi K et al. Pluripotency governed by Sox2 via regulation of Oct3/4 expression in mouse embryonic stem cells. Nat cell Biol 2007; 9: 625-635.

36. Okumura-Nakanishi S, Saito M, Niwa H, Ishikawa F. Oct-3/4 and Sox2 regulate Oct-3/4 gene in embryonic stem cells. J Biol Chem 2005; 280: 5307-5317.

37. Kalmar T, Lim C, Hayward P, Munoz-Descalzo S, Nichols J, Garcia-Ojalvo J et al. Regulated fluctuations in nanog expression mediate cell fate decisions in embryonic stem cells. PLoS Biology 2009; 7: e1000149.

38. Anneren C, Cowan CA, Melton DA. The Src family of tyrosine kinases is important for embryonic stem cell self-renewal. J Biol Chem 2004; 279: 31590-31598.

39. Rubin R, Arzumanyan A, Soliera AR, Ross B, Peruzzi F, Prisco M. Insulin receptor substrate (IRS)-1 regulates murine embryonic stem (mES) cells self-renewal. J Cell Physiol 2007; 213: 445-453.

40. Nott A, Riccio A. Nitric oxide-mediated epigenetic mechanisms in developing neurons. Cell cycle (Georgetown, Tex) 2009; 8: 725-730.

Cell Death and Disease is an open-access journal published by Nature Publishing Group. This work is licensed under the Creative Commons Attribution-Noncommercial-No Derivative Works 3.0 Unported License. To view a copy of this license, visit http://creativecommons.org/licenses/by-nc-nd/3.0/

\section{Supplementary Information accompanies the paper on Cell Death and Disease website (http://www.nature.com/cddis)}

